\title{
Cardioprotective effects of erythropoietin in diabetic rats determined by CD34 and vascular endothelial growth factor levels
}

\author{
Didem Melis Oztas ${ }^{1,2}$, Mert Meric ${ }^{1}$, Metin Onur Beyaz ${ }^{3}$, Mehmet Akif Onalan ${ }^{4}$, Kivilcim Sonmez \\ Kerem Oter ${ }^{5}$, Sedat Ziyade ${ }^{6}$, Beyhan Omer ${ }^{7}$ Ufuk Alpagut ${ }^{1}$, Murat Ugurlucan ${ }^{3}$
}

\author{
'Department of Cardiovascular Surgery, Istanbul Medical Faculty, Istanbul University, \\ Istanbul, Turkey \\ ${ }^{2}$ Cardiovascular Surgery Clinic, Bagcilar Education and Research Hospital, Istanbul, \\ Turkey \\ ${ }^{3}$ Department of Cardiovascular Surgery, Medical Faculty, Medipol University, Istanbul, \\ Turkey \\ ${ }^{4}$ Department of Cardiovascular Surgery, Acibadem Mehmet Ali Aydinlar University, \\ Atakent Hospital, Istanbul, Turkey \\ ${ }^{5}$ Veterinary Faculty, University-Cerrahpasa, Istanbul, Turkey \\ ${ }^{6}$ Department of Thoracic Surgery, Bezmialem University, Istanbul, Turkey \\ ${ }^{7}$ Department of Biochemistry, Medical Faculty, Istanbul University, Istanbul Turkey
}

Submitted: 1 October 2019

Accepted: 24 October 2019

Arch Med Sci Atheroscler Dis 2020; 5: e1-e12

DOI: https://doi.org/10.5114/amsad.2020.92346

Copyright $\odot 2020$ Termedia \& Banach

\section{Abstract}

Introduction: In this study, the effects of diabetes mellitus on the cardiovascular system were investigated by assessing the stem cell levels in serum and heart and compared with the normal population. Additionally, efficacy of erythropoietin, which is known to increase stem cells, was studied in diabetic rats.

Material and methods: Twenty-five male Sprague Dawley rats were divided into three groups as a control group (group 1), diabetic group (group 2) and erythropoietin induced diabetic group (group 3). A diabetes model was created with streptozocin. In group 3 rats received $3000 \mathrm{U} / \mathrm{kg}$ of erythropoietin. At the end of 1 month blood reticulocyte levels, degree of tissue fibrosis and immunohistochemical assessment of reliable stem cell markers, CD34 and vascular endothelial growth factor (VEGF), were analyzed.

Results: The increase in the blood glucose levels resulted in a significant decrease in reticulocyte levels in group 2. The increase in blood glucose levels resulted in a statistically significant increase in tissue level of fibrosis, CD34 and VEGF. When the rats in groups 1 and 2 were compared, the fibrosis, CD34 and VEGF levels were found to increase significantly. When group 2 and group 3 were compared, the amount of fibrosis was lower and the levels of CD34 and VEGF were significantly higher in group 3 than group 2.

Conclusions: The results of our study indicated that the amount of CD34 and VEGF which function in cellular protection and tissue regeneration may be enhanced with safely applicable erythropoietin leading to increase in reticulocyte levels in serum, and CD34 and VEGF levels in right atrium, right ventricle, left atrium, and left ventricle as a protective mechanism in diabetic rats.

Key words: cardioprotective effect mechanisms, erythropoietin, diabetes mellitus.
Corresponding author:

Assoc. Prof. Murat Ugurlucan

TEM Avrupa Otoyolu

Goztepe Cikisi,

No. 1, 34214 Bagcilar

Istanbul, Turkey.

Phone: +90530 8251122

Fax: +90 2125212377

E-mail: didem_mls@hotmail.

com 


\section{Introduction}

Diabetes mellitus (DM) is a complex metabolic syndrome characterized by hyperglycemia, that increases the risk of microvascular and macrovascular diseases [1]. It is one of the major causes of morbidity and mortality with its complications affecting many people through out the world.

Epidemiological studies have shown that diabetes mellitus is an independent risk factor for cardiovascular diseases. The risk of death due to coronary artery disease is high in diabetic patients compared with non-diabetic patients. Furthermore, the risk of mortality in patients with known diabetes mellitus and who are not clinically diagnosed with coronary artery disease is similar to non-diabetic patients who previously experienced myocardial infarction [2]. Hyperglycemia directly causes endothelial dysfunction and is frequently associated with cardiovascular diseases $[3,4]$. The development of atherosclerosis occurs at an earlier age in diabetic patients and rapidly progresses [5].

It is known that myocardial cells lack the ability to regenerate or have very little regeneration capacity. However, regenerative cells have been detected in the myocardium and have given rise to the idea that these cells may be manipulated. Stem cells, which are the building blocks of all living subjects, rest in different regions of the tissues. They are programmed to increase tissue regeneration, especially in the case of tissue loss or damage [6].

Although studies have shown that stem cells reduce cardiac damage caused by ischemia [6-8], the most important limiting factor in these studies is that a determined methodology has not been established. It has been shown that angiographic methods or intraoperative injected stem cells after myocardial infarction reduced ischemic cardiac damage and affect myocardial functions positively $[9,10]$. Erythropoietin is a glycoprotein molecule which is synthesized in the case of hypoxia. In preclinical studies, it has been reported that the erythropoietin molecule has significant tissue protective effects with mechanisms such as apoptosis inhibition, vascular restoration, reduction of inflammatory response and increased tissue function in cardiac, neuronal, retinal and renal ischemic injury models $[11,12]$. Recent studies have shown that erythropoietin (EPO) has tissue-protecting effects in the heart by increasing vascular endothelial growth factor (VEGF) expression and alleviating myocardial fibrosis in ischemia models [13].

Many studies using stem cells have ignored diabetic patients and usually the effects of diabetes mellitus on endothelial and coronary arteries have been mostly emphasized $[14,15]$. In this study, we aimed to evaluate the effects of erythropoietin on stem cell synthesis and tissue regeneration in the context of damaged tissues.

\section{Material and methods}

\section{Research plan}

The study was carried out at the Istanbul University Experimental Medicine and Research Institute. Histopathological examinations were performed at Istanbul University Cerrahpasa Veterinary Faculty, Department of Pathology and biochemical investigations were performed at Istanbul University Medical Faculty. The necessary approval was obtained from the Experimental Medicine Ethics Committee of the Experimental Medicine and Research Institute of Istanbul University (Ethics Committee no: 27/2018).

In the study 25 adult Sprague Dawley rats (approximately 3 months) fed with normal food at a suitable weight of 350-400 g were used. The rats were divided into three groups as the control group (group 1), diabetic group (group 2) and erythropoietin-induced diabetic group (group 3).

\section{Measurement methods}

In all rats, blood glucose levels were measured in a drop blood taken from the tail vein after $6 \mathrm{~h}$ of fasting before the experiment and rats with a blood glucose level $\geq 110 \mathrm{mg} / \mathrm{dl}$ were not included in the study. Additionally, mean basal blood glucose level was determined. A MediSense Precision Q.I.D glucometer and MediSense Sensor Electrodes (Abbott Laboratories Warszawa, Poland) were used for blood glucose measurement. In group 2 and group 3 rats, following the anesthesia achieved by $40 \mathrm{mg} / \mathrm{kg}$ sodium pentothal, intraperitoneal $50 \mathrm{mg} / \mathrm{kg}$ streptozocin (STZ) was given and irreversible pancreatic damage was induced. Following the second day of streptozocin administration, blood drops taken from the tail vein after $6 \mathrm{~h}$ of fasting were measured, and blood glucose levels were higher than $300 \mathrm{mg} / \mathrm{dl}$ in all rats.

The rats were kept alive for 1 month in order to observe the possible cardiovascular complications of diabetes. Blood glucose re-measurements were not required, and clinical findings of diabetes such as polyuria and polydipsia were monitored. During the follow-up period, 2 rats died in the diabetes group probably due to the side effects of diabetes mellitus.

In group 3, stem cell synthesis was induced by administering $3000 \mathrm{U} / \mathrm{kg}$ erythropoietin 2 times intraperitoneally on the $24^{\text {th }}$ and $27^{\text {th }}$ days before rats were sacrificed.

At the end of 1 month, rats in all groups were euthanized with high dose anesthesia $(90 \mathrm{mg} /$ kg pentothal) and blood was drawn. In order to evaluate the stem cell levels, blood samples were 
obtained from the rats and the heart was removed en bloc for adequate sampling of the right atrium, right ventricle, left atrium and left ventricular tissues (Figure 1).

\section{Tissue fibrosis, CD34 and VEGF level measurement}

Heart tissues were fixed in 10\% formalin. Right atrium, right ventricle, left atrium and left ventricular biopsies were taken from each heart tissue after fixation. In the pathology laboratory, the specimen block was prepared following alcohol, xylene and paraffin applications on the tissues. By using an automatic microtome, 4 micron thick sections were taken from each of the paraffin-embedded tissues; and hematoxylin-eosin, mason trichrome staining and immunohistochemical studies were performed with appropriate methods and suitable antigens according to the manufacturers' protocols, which were previously explained in detail in many studies in the literature.

The results were evaluated for each category from 0 to 3: 0 - no staining, 1 - poor staining (10\% staining), 2 - moderate staining (10-50\% staining), 3 - severe staining (more than 50\% staining).

\section{Statistical analysis}

Statistical analysis was performed using the SPSS (SPSS for Windows, SPSS Inc, Chicago, IL, US) 15.0 program package. Variables were expressed as mean \pm standard deviation. Non-parametric methods were used in the statistical evaluation of the data. Kruskal-Wallis variance analysis was used to evaluate the difference between the groups. The Mann-Whitney $U$ test with Bonferroni correction was used for determination of the difference between groups. Correlation analysis of the data was performed by Spearman rank correlation analysis. A $p$-value of less than 0.05 was considered statistically significant.

\section{Results}

The mean blood glucose level was measured before the experiment in all rats and found to be $72.80 \pm 11.516 \mathrm{mg} / \mathrm{dl}$. After the administration of streptozocin, mean blood glucose level was $358.70 \pm 44.749 \mathrm{mg} / \mathrm{dl}$ on the $2^{\text {nd }}$ day and 382.80 $\pm 34.941 \mathrm{mg} / \mathrm{dl}$ at the end of the $1^{\text {st }}$ week. According to the Spearman rank correlation analysis, the rho values were evaluated as follows: $0.00-0.24$ : poor correlation, 0.25-0.49: moderate correlation, 0.50-0.74: strong correlation, $0.75-1.00$ : very strong correlation.

The increase in blood sugar resulted in a statistically significant decrease in reticulocyte levels in group 2 and a statistically significant increase in tissue levels of CD34 and VEGF in group 2 and

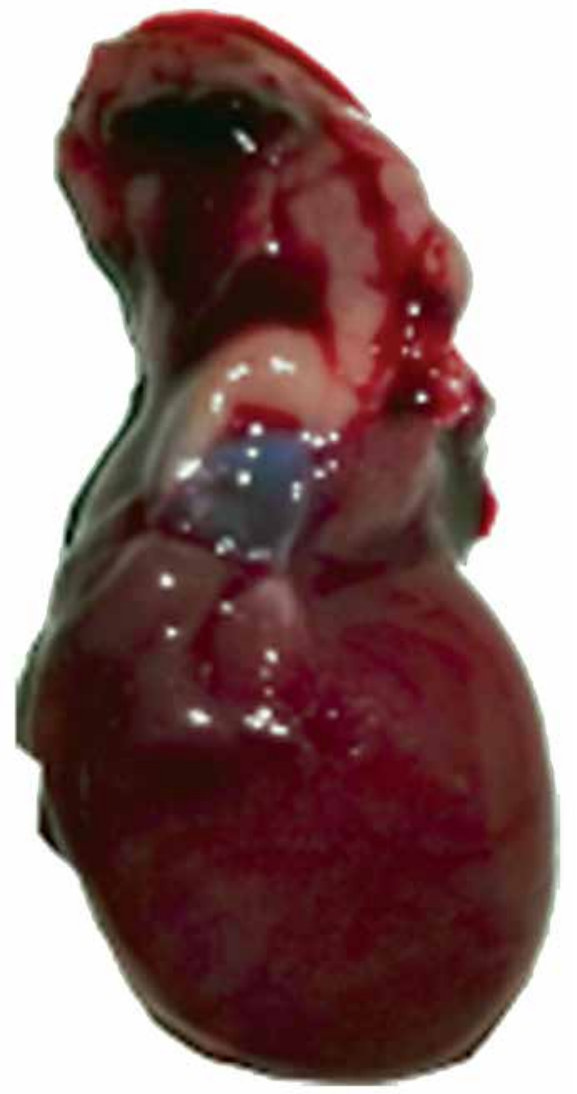

Figure 1. En bloc cardiac tissue

group 3. There was a strong positive and statistically significant correlation only between right atrium CD34 levels and serum reticulocyte levels (rho $=0.701, p=0.024$ ); however, such a correlation was not observed for the left atrium, left ventricle and right ventricle, unexpectedly.

In the control group, diabetic group and erythropoietin-induced diabetic group, the difference in right atrium, right ventricle, left atrium and left ventricular fibrosis was found to be strongly significant when comparing the groups with each other. According to the results of the analysis, the differences between the fibrosis levels of the 3 groups in the right atrial tissue were significant $\left(\chi_{\text {K-W }}^{2}=\right.$ 8.858 , SD $=2, p=0.012$ ). No significant difference was found between group 1 and group 3, and group 2 and group 3. There was a significant difference between group 1 and group 2 (Table I). This significant difference was proved to be due to less fibrosis in group 1.

The difference between the fibrosis levels of the 3 groups in the right ventricular tissue $\left(\chi_{\text {K-W }}^{2}=\right.$ $13.200, \mathrm{SD}=2, p=0.001)$, left atrial tissues $\left(\chi_{\mathrm{K}-\mathrm{w}}^{2}=\right.$ $14.883, \mathrm{SD}=2, p=0.001)$ and left ventricular tissues $\left(\chi_{\mathrm{K}-\mathrm{W}}^{2}=13.520, \mathrm{SD}=2, p=0.001\right)$ were statistically significant. The differences between group 1 and group 2, and group 2 and group 3 
were significant while the difference between group 1 and group 3 was not significant (Tables II-IV). This difference was attributed to increased fibrosis in group 2 (Figure 2) and minimal fibrosis in group 1 and attenuated fibrosis in group 3 with erythropoietin injection.

When the right atrial, right ventricular, left atrial and left ventricular CD34 were compared

Table I. Statistical analysis of fibrosis, CD34 and VEGF levels in right atrium of control, diabetic and diabetic rats induced with erythropoietin. A - Descriptive statistics, B - Kruskal-Wallis test, test statistics, C - Mann-Whitney $1 \& 2$ test

A

\begin{tabular}{|c|c|c|c|c|c|c|}
\hline Group & Parameter & $N$ & Minimum & Maximum & Mean & Std. deviation \\
\hline \multirow[t]{6}{*}{ Control } & Reticulocyte\% & 5 & 1.20 & 1.49 & 1.3300 & 0.11790 \\
\hline & Glucose & 5 & 77 & 100 & 90.00 & 10.700 \\
\hline & CD34 & 5 & 1 & 2 & 1.20 & 0.447 \\
\hline & VEGF & 5 & 1 & 2 & 1.20 & 0.447 \\
\hline & Fibrosis & 5 & 1 & 2 & 1.20 & 0.447 \\
\hline & Valid N (listwise) & 5 & & & & \\
\hline \multirow[t]{6}{*}{ Diabetic } & Reticulocyte\% & 8 & 1.30 & 2.80 & 1.9250 & 0.53918 \\
\hline & Glucose & 8 & 270 & 494 & 397.50 & 71.841 \\
\hline & CD34 & 8 & 1 & 3 & 1.62 & 0.744 \\
\hline & VEGF & 8 & 1 & 3 & 1.88 & 0.641 \\
\hline & Fibrosis & 8 & 1 & 3 & 2.50 & 0.756 \\
\hline & Valid N (listwise) & 8 & & & & \\
\hline \multirow[t]{6}{*}{ Erythropoietin } & Reticulocyte\% & 10 & 12.00 & 28.00 & 20.9000 & 5.70477 \\
\hline & Glucose & 10 & 350 & 450 & 410.70 & 31.609 \\
\hline & CD34 & 10 & 2 & 3 & 2.80 & 0.422 \\
\hline & VEGF & 10 & 2 & 3 & 2.70 & 0.483 \\
\hline & Fibrosis & 10 & 1 & 3 & 2.10 & 0.568 \\
\hline & Valid N (listwise) & 10 & & & & \\
\hline
\end{tabular}

B

\begin{tabular}{|lccccc|}
\hline Parameter & Reticulocyte $\%$ & Glucose & CD34 & VEGF & Fibrosis \\
\hline$\chi^{2}$ & 18.319 & 11.267 & 13.892 & 12.727 & 8.858 \\
\hline $\mathrm{d} f$ & 2 & 2 & 2 & 2 & 0.002 \\
\hline Asymp. sig. & 0.000 & 0.004 & 0.001 & 0.002 \\
\hline
\end{tabular}

Kruskal-Wallis test, grouping variable: group.

C

\begin{tabular}{|c|c|c|c|c|c|}
\hline Parameter & Reticulocyte\% & Glucose & CD34 & VEGF & Fibrosis \\
\hline Mann-Whitney $U$ & 3.000 & 0.000 & 13.500 & 8.500 & 4.000 \\
\hline Wilcoxon W & 18.000 & 15.000 & 28.500 & 23.500 & 19.000 \\
\hline$Z$ & -2.492 & -2.928 & -1.105 & -1.873 & -2.498 \\
\hline Asymp. sig. (2-tailed) & 0.013 & 0.003 & 0.269 & 0.061 & 0.012 \\
\hline ExactSig. [2*(1-tailed sig.)] & $0.011^{\mathrm{a}}$ & $0.002^{a}$ & $0.354^{a}$ & $0.093^{a}$ & $0.019^{a}$ \\
\hline
\end{tabular}

${ }^{a}$ Not corrected for ties. 
Table I. Cont. D - Mann-Whitney $2 \& 3$ test, E - Mann-Whitney $1 \& 3$ test

D

\begin{tabular}{|lccccc|}
\hline Parameter & Reticulocyte\% & Glucose & CD34 & VEGF & Fibrosis \\
\hline Mann-Whitney U & 0.000 & 38.500 & 9.000 & 14.000 & 25.500 \\
\hline Wilcoxon $W$ & 36.000 & 74.500 & 45.000 & 50.000 & 80.500 \\
\hline$Z$ & -3.560 & -.133 & -2.996 & -2.542 & -1.425 \\
\hline Asymp. sig. (2-tailed) & 0.000 & 0.894 & 0.003 & 0.011 & 0.154 \\
\hline ExactSig. [2*(1-tailed Sig.)] & $0.000^{\mathrm{a}}$ & $0.897^{\mathrm{a}}$ & $0.004^{\mathrm{a}}$ & $0.021^{\mathrm{a}}$ & $0.203^{\mathrm{a}}$ \\
\hline
\end{tabular}

${ }^{a}$ Not corrected for ties.

E

\begin{tabular}{|lccccc|}
\hline Parameter & Reticulocyte $\%$ & Glucose & CD34 & VEGF & Fibrosis \\
\hline Mann-Whitney $U$ & 0.000 & 38.500 & 9.000 & 14.000 & 25.500 \\
\hline Wilcoxon W & 36.000 & 74.500 & 45.000 & 50.000 & -2.542 \\
\hline$Z$ & -3.560 & -.133 & -2.996 & 0.500 & -1.425 \\
\hline Asymp. sig. (2-tailed) & 0.000 & 0.894 & 0.003 & 0.154 \\
\hline ExactSig. [2*(1-tailed Sig.)] & $0.000^{\text {a }}$ & $0.897^{\text {a }}$ & $0.004^{\text {a }}$ & $0.021^{\text {a }}$ & $0.203^{\text {a }}$ \\
\hline
\end{tabular}

${ }^{a}$ Not corrected for ties.

Table II. Statistical analysis of fibrosis, CD34 and VEGF levels in right ventricle of control, diabetic and diabetic rats induced with erythropoietin. A - Descriptive statistics

A

\begin{tabular}{|c|c|c|c|c|c|c|}
\hline Group & Parameter & $N$ & Minimum & Maximum & Mean & Std. deviation \\
\hline \multirow[t]{6}{*}{ Control } & Reticulocyte\% & 5 & 1.20 & 1.49 & 1.3300 & 0.11790 \\
\hline & Glucose & 5 & 77 & 100 & 90.00 & 10.700 \\
\hline & CD34 & 5 & 1 & 2 & 1.40 & 0.548 \\
\hline & VEGF & 5 & 1 & 2 & 1.20 & 0.447 \\
\hline & Fibrosis & 5 & 1 & 1 & 1.00 & 0.000 \\
\hline & Valid N (listwise) & 5 & & & & \\
\hline \multirow[t]{6}{*}{ Diabetic } & Reticulocyte\% & 8 & 1.30 & 2.80 & 1.9250 & 0.53918 \\
\hline & Glucose & 8 & 270 & 494 & 397.50 & 71.841 \\
\hline & CD34 & 8 & 1 & 3 & 1.88 & 0.835 \\
\hline & VEGF & 8 & 1 & 3 & 2.00 & 0.756 \\
\hline & Fibrosis & 8 & 2 & 3 & 2.75 & 0.463 \\
\hline & Valid N (listwise) & 8 & & & & \\
\hline \multirow[t]{6}{*}{ Erythropoietin } & Reticulocyte\% & 10 & 12.00 & 28.00 & 20.9000 & 5.70477 \\
\hline & Glucose & 10 & 350 & 450 & 410.70 & 31.609 \\
\hline & CD34 & 10 & 1 & 3 & 2.60 & 0.699 \\
\hline & VEGF & 10 & 2 & 3 & 2.60 & 0.516 \\
\hline & Fibrosis & 10 & 1 & 3 & 1.90 & 0.738 \\
\hline & Valid N (listwise) & 10 & & & & \\
\hline
\end{tabular}


Table II. Cont. B - Kruskal-Wallis test, test statistics, C - Mann-Whitney $1 \& 2$ test, D - Mann-Whitney $1 \& 3$ test, E - Mann-Whitney $2 \& 3$ test

B

\begin{tabular}{|lccccc|}
\hline Parameter & Reticulocyte $\%$ & Glucose & CD34 & VEGF & Fibrosis \\
\hline$\chi^{2}$ & 18.319 & 11.267 & 7.573 & 10.294 & 13.200 \\
\hline d $f$ & 2 & 2 & 2 & 2 & 2 \\
\hline Asymp. sig. & 0.000 & 0.004 & 0.023 & 0.006 & 0.001 \\
\hline
\end{tabular}

C

\begin{tabular}{|lccccc|}
\hline Parameter & Reticulocyte\% & Glucose & CD34 & VEGF & Fibrosis \\
\hline Mann-Whitney $U$ & 3.000 & 0.000 & 13.500 & 8.000 & 0.000 \\
\hline Wilcoxon $W$ & 18.000 & 15.000 & 28.500 & 23.000 & 15.000 \\
\hline$Z$ & -2.492 & -2.928 & -1.034 & -1.910 & -3.183 \\
\hline Asymp. sig. (2-tailed) & 0.013 & 0.003 & 0.301 & 0.056 & 0.001 \\
\hline ExactSig. [2*(1-tailed sig.)] & $0.011^{\mathrm{a}}$ & $0.002^{\mathrm{a}}$ & $0.354^{\mathrm{a}}$ & $0.093^{\mathrm{a}}$ & $0.002^{\mathrm{a}}$ \\
\hline
\end{tabular}

${ }^{a}$ Not corrected for ties.

D

\begin{tabular}{|lccccc|}
\hline Parameter & Reticulocyte\% & Glucose & CD34 & VEGF & Fibrosis \\
\hline Mann-Whitney $U$ & 0.000 & 0.000 & 5.500 & 2.000 & 7.500 \\
\hline Wilcoxon $W$ & 15.000 & 15.000 & 20.500 & 17.000 & 22.500 \\
\hline$Z$ & -3.067 & -3.062 & -2.569 & -2.996 & -2.378 \\
\hline Asymp. sig. (2-tailed) & 0.002 & 0.002 & 0.010 & 0.003 & 0.017 \\
\hline ExactSig. [2*(1-tailed sig.)] & $0.001^{\mathrm{a}}$ & $0.001^{\mathrm{a}}$ & $0.013^{\mathrm{a}}$ & $0.003^{\mathrm{a}}$ & $0.028^{\mathrm{a}}$ \\
\hline
\end{tabular}

${ }^{a}$ Not corrected for ties.

E

\begin{tabular}{|c|c|c|c|c|c|}
\hline Parameter & Reticulocyte\% & Glucose & CD34 & VEGF & Fibrosis \\
\hline Mann-Whitney $U$ & 0.000 & 38.500 & 20.500 & 22.000 & 15.000 \\
\hline Wilcoxon W & 36.000 & 74.500 & 56.500 & 58.000 & 70.000 \\
\hline$Z$ & -3.560 & -0.133 & -1.885 & -1.760 & -2.407 \\
\hline Asymp. sig. (2-tailed) & 0.000 & 0.894 & 0.059 & 0.078 & 0.016 \\
\hline ExactSig. [2*(1-tailed sig.)] & $0.000^{\mathrm{a}}$ & $0.897^{\mathrm{a}}$ & $0.083^{a}$ & $0.122^{\mathrm{a}}$ & $0.027^{a}$ \\
\hline
\end{tabular}

${ }^{a}$ Not corrected for ties.

between the control group, diabetic group and erythropoietin-induced diabetic group, the difference was found to be highly significant. According to the results of the analysis, the differences between the CD34 levels of the 3 groups in the right atrial tissue $\left(\chi_{\mathrm{K}-\mathrm{W}}^{2}=13.892, \mathrm{SD}=2, p=0.001\right)$ and left atrial tissue $\left(\chi_{\text {K-w }}^{2}=11.130, \mathrm{SD}=2, p=0.004\right)$ were statistically significant. There was a significant difference between group 1 and group 3, and group 2 and group 3. No significant difference was found between group 1 and group 2 (Tables I, III).
The differences between the CD34 levels of the 3 groups in the right ventricular tissue $\left(\chi_{\mathrm{K}-\mathrm{w}}^{2}=\right.$ $7.573, \mathrm{SD}=2, p=0.023)$ and left ventricular tissue $\left(\chi_{\mathrm{K}-\mathrm{W}}^{2}=9.855, \mathrm{SD}=2, p=0.007\right)$ were statistically significant. A significant difference was found between group 1 and group 3, and no significant difference was found between group 1 and group 2, and group 2 and group 3 (Tables II, IV). The significant differences between the CD34 levels of different tissues were proven to be due to the high amount of CD34 in group 3 (Figure 3). 
Table III. Statistical analysis of fibrosis, CD34 and VEGF levels in left atrium of control, diabetic and diabetic rats induced with erythropoietin. A - Descriptive statistics, B - Kruskal-Wallis test, test statistics, C - Mann-Whitney $1 \& 2$ test

A

\begin{tabular}{|c|c|c|c|c|c|c|}
\hline Group & Parameter & $N$ & Minimum & Maximum & Mean & Std. deviation \\
\hline \multirow[t]{6}{*}{ Control } & Reticulocyte\% & 5 & 1.20 & 1.49 & 1.3300 & 0.11790 \\
\hline & Glucose & 5 & 77 & 100 & 90.00 & 10.700 \\
\hline & CD34 & 5 & 1 & 2 & 1.40 & 0.548 \\
\hline & VEGF & 5 & 1 & 2 & 1.20 & 0.447 \\
\hline & Fibrosis & 5 & 1 & 2 & 1.20 & 0.447 \\
\hline & Valid $N$ (listwise) & 5 & & & & \\
\hline \multirow[t]{6}{*}{ Diabetic } & Reticulocyte\% & 8 & 1.30 & 2.80 & 1.9250 & 0.53918 \\
\hline & Glucose & 8 & 270 & 494 & 397.50 & 71.841 \\
\hline & CD34 & 8 & 1 & 3 & 1.88 & 0.835 \\
\hline & VEGF & 8 & 1 & 2 & 1.75 & 0.463 \\
\hline & Fibrosis & 8 & 2 & 3 & 2.87 & 0.354 \\
\hline & Valid $N$ (listwise) & 8 & & & & \\
\hline \multirow[t]{6}{*}{ Erythropoietin } & Reticulocyte\% & 10 & 12.00 & 28.00 & 20.9000 & 5.70477 \\
\hline & Glucose & 10 & 350 & 450 & 410.70 & 31.609 \\
\hline & CD34 & 10 & 2 & 3 & 2.80 & 0.422 \\
\hline & VEGF & 10 & 2 & 3 & 2.80 & 0.422 \\
\hline & Fibrosis & 10 & 1 & 3 & 1.90 & 0.568 \\
\hline & Valid N (listwise) & 10 & & & & \\
\hline
\end{tabular}

B

\begin{tabular}{|lccccc|}
\hline Parameter & Reticulocyte $\%$ & Glucose & CD34 & VEGF & Fibrosis \\
\hline$\chi^{2}$ & 18.319 & 11.267 & 11.130 & 15.961 & 2 \\
\hline $\mathrm{d} f$ & 2 & 2 & 2 & 0.000 & 0.001 \\
\hline Asymp. sig. & 0.000 & 0.004 & 0.004 & 0.000 \\
\hline
\end{tabular}

C

\begin{tabular}{|lccccc|}
\hline Parameter & Reticulocyte\% & Glucose & CD34 & VEGF & Fibrosis \\
\hline Mann-Whitney U & 3.000 & 0.000 & 13.500 & 9.000 & 15.500 \\
\hline Wilcoxon W & 18.000 & 15.000 & 28.500 & 24.000 & -1.859 \\
\hline Z & -2.492 & -2.928 & -1.034 & 0.063 & 0.002 \\
\hline Asymp. sig. (2-tailed) & 0.013 & 0.003 & 0.301 & $0.127^{\mathrm{a}}$ & $0.002^{\mathrm{a}}$ \\
\hline ExactSig. [2*(1-tailed sig.)] & $0.011^{\mathrm{a}}$ & $0.002^{\mathrm{a}}$ & $0.354^{\mathrm{a}}$ & & \\
\hline
\end{tabular}

${ }^{a}$ Not corrected for ties. 
Table III. Cont. D - Mann-Whitney $1 \& 3$ test, E - Mann-Whitney $2 \& 3$ test

D

\begin{tabular}{|lccccc|}
\hline Parameter & Reticulocyte\% & Glucose & CD34 & VEGF & Fibrosis \\
\hline Mann-Whitney $U$ & 0.000 & 0.000 & 2.000 & 1.000 & 9.500 \\
\hline Wilcoxon $W$ & 15.000 & 15.000 & 17.000 & 16.000 & 24.500 \\
\hline$Z$ & -3.067 & -3.062 & -3.101 & -3.236 & -2.139 \\
\hline Asymp. sig. (2-tailed) & 0.002 & 0.002 & 0.002 & 0.001 & 0.032 \\
\hline ExactSig. [2*(1-tailed sig.)] & $0.001^{\mathrm{a}}$ & $0.001^{\mathrm{a}}$ & $0.003^{\mathrm{a}}$ & $0.001^{\mathrm{a}}$ & $0.055^{\mathrm{a}}$ \\
\hline
\end{tabular}

${ }^{a}$ Not corrected for ties.

$\mathrm{E}$

\begin{tabular}{|c|c|c|c|c|c|}
\hline Parameter & Reticulocyte\% & Glucose & CD34 & VEGF & Fibrosis \\
\hline Mann-Whitney $U$ & 0.000 & 38.500 & 15.000 & 6.000 & 8.000 \\
\hline Wilcoxon $W$ & 36.000 & 74.500 & 51.000 & 42.000 & 63.000 \\
\hline$Z$ & -3.560 & -0.133 & -2.476 & -3.325 & -3.129 \\
\hline Asymp. sig. (2-tailed) & 0.000 & 0.894 & 0.013 & 0.001 & 0.002 \\
\hline ExactSig. [2*(1-tailed sig.)] & $0.000^{\mathrm{a}}$ & $0.897^{a}$ & $0.027^{a}$ & $0.001^{\mathrm{a}}$ & $0.003^{a}$ \\
\hline
\end{tabular}

${ }^{a}$ Not corrected for ties.

Table IV. Statistical analysis of fibrosis, CD34 and VEGF levels in left ventricle of control, diabetic and diabetic rats induced with erythropoietin. A - Descriptive statistics

A

\begin{tabular}{|c|c|c|c|c|c|c|}
\hline Group & Parameter & $N$ & Minimum & Maximum & Mean & Std. deviation \\
\hline \multirow[t]{6}{*}{ Control } & Reticulocyte\% & 5 & 1.20 & 1.49 & 1.3300 & 0.11790 \\
\hline & Glucose & 5 & 77 & 100 & 90.00 & 10.700 \\
\hline & CD34 & 5 & 1 & 2 & 1.40 & 0.548 \\
\hline & VEGF & 5 & 1 & 2 & 1.20 & 0.447 \\
\hline & Fibrosis & 5 & 1 & 2 & 1.20 & 0.447 \\
\hline & Valid N (listwise) & 5 & & & & \\
\hline \multirow[t]{6}{*}{ Diabetic } & Reticulocyte\% & 8 & 1.30 & 2.80 & 1.9250 & 0.53918 \\
\hline & Glucose & 8 & 270 & 494 & 397.50 & 71.841 \\
\hline & CD34 & 8 & 1 & 3 & 2.00 & 0.756 \\
\hline & VEGF & 8 & 1 & 2 & 1.75 & 0.463 \\
\hline & Fibrosis & 8 & 2 & 3 & 2.87 & 0.354 \\
\hline & Valid N (listwise) & 8 & & & & \\
\hline \multirow[t]{6}{*}{ Erythropoietin } & Reticulocyte\% & 10 & 12.00 & 28.00 & 20.9000 & 5.70477 \\
\hline & Glucose & 10 & 350 & 450 & 410.70 & 31.609 \\
\hline & CD34 & 10 & 2 & 3 & 2.70 & 0.483 \\
\hline & VEGF & 10 & 2 & 3 & 2.70 & 0.483 \\
\hline & Fibrosis & 10 & 1 & 3 & 2.00 & 0.667 \\
\hline & Valid $N$ (listwise) & 10 & & & & \\
\hline
\end{tabular}


Table IV. Cont. B - Kruskal-Wallis test, test statistics, C - Mann-Whitney $1 \& 2$ test, D - Mann-Whitney $1 \& 3$ test, E - Mann-Whitney $2 \& 3$ test

B

\begin{tabular}{|lccccc|}
\hline Parameter & Reticulocyte\% & Glucose & CD34 & VEGF & Fibrosis \\
\hline$\chi^{2}$ & 18.319 & 11.267 & 9.855 & 14.561 & 13.520 \\
\hline $\mathrm{d} f$ & 2 & 2 & 2 & 2 & 2 \\
\hline Asymp. sig. & 0.000 & 0.004 & 0.007 & 0.001 & 0.001 \\
\hline
\end{tabular}

C

\begin{tabular}{|lccccc|}
\hline Parameter & Reticulocyte\% & Glucose & CD34 & VEGF & Fibrosis \\
\hline Mann-Whitney $U$ & 3.000 & 0.000 & 11.000 & 9.000 & 0.500 \\
\hline Wilcoxon $W$ & 18.000 & 15.000 & 26.000 & 24.000 & 15.500 \\
\hline$Z$ & -2.492 & -2.928 & -1.432 & -1.859 & -3.160 \\
\hline Asymp. sig. (2-tailed) & 0.013 & 0.003 & 0.152 & 0.063 & 0.002 \\
\hline ExactSig. [2*(1-tailed sig.) & $0.011^{\mathrm{a}}$ & $0.002^{\mathrm{a}}$ & $0.222^{\mathrm{a}}$ & $0.127^{\mathrm{a}}$ & $0.002^{\mathrm{a}}$ \\
\hline
\end{tabular}

${ }^{a}$ Not corrected for ties.

D

\begin{tabular}{|lccccc|}
\hline Parameter & Reticulocyte\% & Glucose & CD34 & VEGF & Fibrosis \\
\hline Mann-Whitney $U$ & 0.000 & 0.000 & 3.000 & 1.500 & 9.000 \\
\hline Wilcoxon $W$ & 15.000 & 15.000 & 18.000 & 16.500 & 24.000 \\
\hline$Z$ & -3.067 & -3.062 & -2.910 & -3.096 & -2.144 \\
\hline Asymp. sig. (2-tailed) & 0.002 & 0.002 & 0.004 & 0.002 & 0.032 \\
\hline ExactSig. [2*(1-tailed sig.)] & $0.001^{\text {a }}$ & $0.001^{\text {a }}$ & $0.005^{\text {a }}$ & $0.001^{\text {a }}$ & $0.055^{\text {a }}$ \\
\hline
\end{tabular}

${ }^{a}$ Not corrected for ties.

E

\begin{tabular}{|lccccc|}
\hline Parameter & Reticulocyte\% & Glucose & CD34 & VEGF & Fibrosis \\
\hline Mann-Whitney U & 0.000 & 38.500 & 19.000 & 9.000 & 67.000 \\
\hline Wilcoxon W & 36.000 & 74.500 & 55.000 & 45.000 & -3.047 \\
\hline Z & -3.560 & -0.133 & -2.064 & 0.002 & 0.006 \\
\hline Asymp. sig. (2-tailed) & 0.000 & 0.894 & 0.039 & $0.004^{\mathrm{a}}$ & $0.012^{\mathrm{a}}$ \\
\hline ExactSig. [2*(1-tailed sig.)] & $0.000^{\mathrm{a}}$ & $0.897^{\mathrm{a}}$ & $0.068^{\mathrm{a}}$ & & \\
\hline
\end{tabular}

${ }^{a}$ Not corrected for ties.

When the right atrium, right ventricle, left atrium and left ventricular VEGF levels were compared in the control group, diabetic group and erythropoietin induced diabetic group, the difference was found to be highly significant. According to the results of the analysis, the difference between the VEGF levels of the 3 groups in the right atrial tissue $\left(\chi_{\mathrm{K}-\mathrm{w}}^{2}=12.727, \mathrm{SD}=2, p=0.002\right)$, and left atrial tissue $\left(\chi_{\mathrm{K}-\mathrm{W}}^{2}=15.961, \mathrm{SD}=2, p<\right.$ $0.001)$ was statistically significant. There was significant difference between group 1 and group 3 and group 2 and group 3, and no significant dif- ference was found between group 1 and group 2 (Tables I, III, IV). The differences between the VEGF levels of the 3 groups in the right ventricular tissue $\left(\chi_{\mathrm{k}-\mathrm{W}}^{2}=10.294, \mathrm{SD}=2, p=0.006\right)$ and left ventricular tissue $\left(\chi_{\mathrm{K}-\mathrm{W}}^{2}=14.561, \mathrm{SD}=2\right.$, $p=0.001)$ were statistically significant. There was a statistically significant difference between group 1 and group 3, and no significant difference was found between group 1 and group 2, and group 2 and group 3 (Table II). This significant difference was proved to be due to the high amount of VEGF in group 3 (Figure 4). 


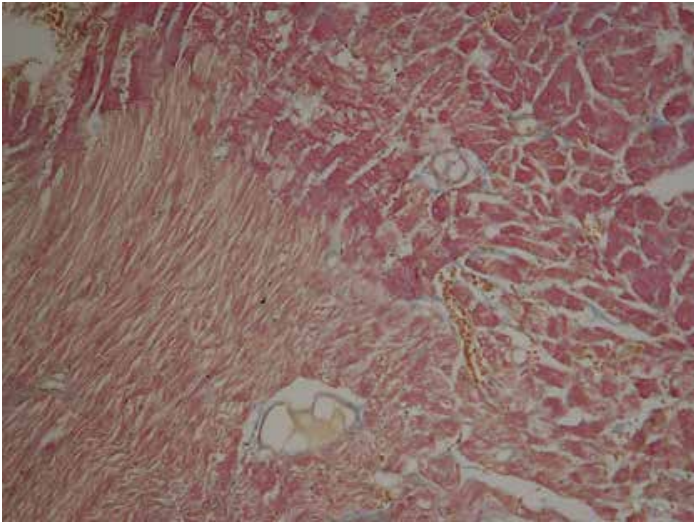

Figure 2. Thin fibrillary connective tissue formation in necrotic tissue by Masson trichrome staining in diabetic group

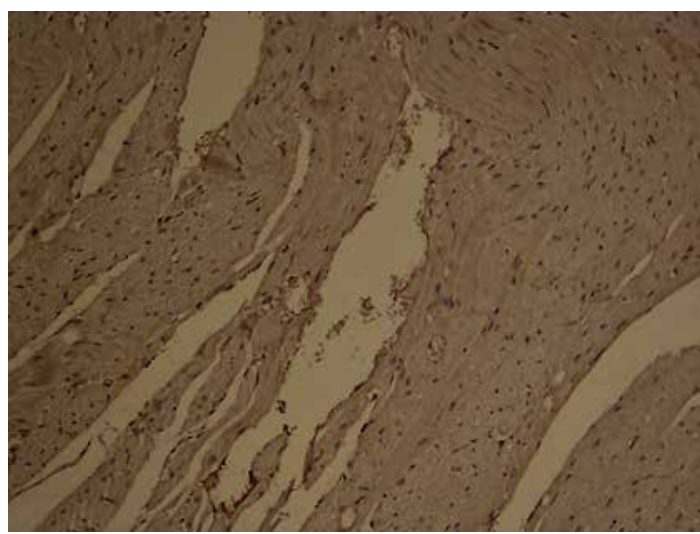

Figure 4. Increased tissue stain with VEGF

\section{Discussion}

In developed western countries, at least half of all deaths are due to cardiovascular diseases and $3 / 4$ of them are related to atherosclerotic coronary artery disease (CAD). It is one of the leading causes of morbidity and mortality [16]. Diabetes mellitus is defined as a complex metabolic syndrome characterized by hyperglycemia, which increases the risk of microvascular and macrovascular disease [1]. Diabetes was closely associated with other risk factors and due to high mortality (46 times) rates in the case of myocardial infarction, it was accepted as a risk equivalent of CAD [4].

Studies on stem cells also attract attention in the field of cardiac surgery. In order to help tissue regeneration in stress conditions such as ischemia, hyperthermia and hypothermia, these cells, which increase in number, may also serve in the defense mechanisms when needed in various stress situations $[17,18]$. Studies have shown that stem cells may reduce cardiac damage caused by ischemia [7, 8].

Erythropoietin is a glycoprotein hormone that is induced by hypoxia that stimulates the proliferation and differentiation of erythroid precursor

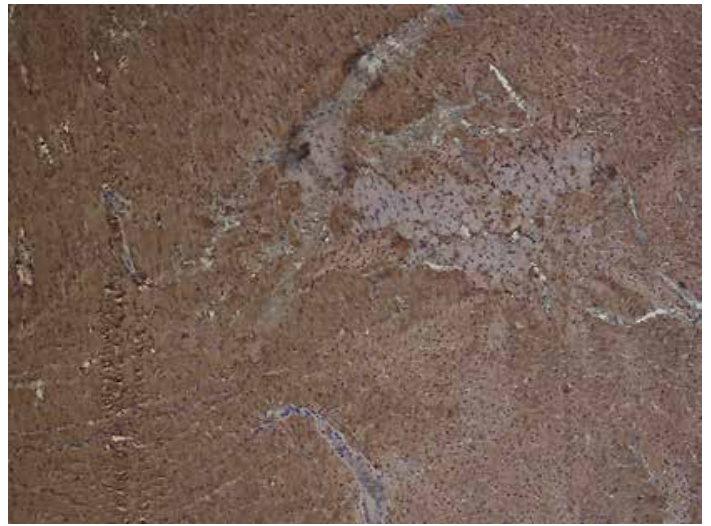

Figure 3. Increased tissue stain with CD34

cells to eliminate the reduced levels of oxygen caused by anemia and hypoxia. Several studies have shown that EPO can induce proliferation cascades in the myocardium [12]. Thus, the idea that EPO may be used as a cytoprotective agent in the cardiovascular system has been born [19, 20]. EPO may also trigger neovascularization, reduce infarct size and provide effective cardiac protection against ischemia-reperfusion injury and chronic heart failure. Erythropoietin protects the myocardium from ischemic injury and promotes re-modeling [11].

Diabetic cardiomyopathy (DCM) is characterized by microvascular pathology and interstitial fibrosis leading to progressive heart failure. Diabetic microvascular complications are thought to be affected by angiogenic factors, including VEGF, in response to both ischemia and hyperglycemia. Hyperglycemia also causes interstitial fibrosis and progressive cardiac dysfunction along with apoptosis and necrosis of cardiomyocytes $[18,20]$. In a study with EPO in DCM-induced rats, it was found that EPO administration can reverse the reshaping of the heart by increasing angiogenesis and weakening interstitial fibrosis without affecting blood sugar [18]. Erythropoietin has been shown to have tissue-protective effects in the heart by increasing VEGF expression and alleviating myocardial fibrosis in ischemia models [19].

Many studies using stem cells have ignored diabetic patients. However, we know that diabetes is a risk factor for many postoperative morbidities which are thought to be caused by differences in stem cell level $[14,15]$. Streptozocin is a pancreatic beta cell toxin that disrupts the ability to produce insulin in animals and has led to the development of the model of diabetic atherosclerosis. This model was first described by Park and resulted in a 5.3-fold plaque increase in the aortic sinus compared to the control group after 6-week follow-up in mice with diabetes induced by streptozocin [21]. Thus, diabetic atherosclerosis, which develops plaque morphology in a similar way to 
human beings, was created in the animal model and it allowed animal experiments in diabetic models.

In this study, we aimed to investigate the effects of diabetes on the direct cardiovascular system tissues and the effect of erythropoietin, which is known to increase stem cells in diabetic rats. In the literature, it is seen that VEGF levels are investigated most frequently and safely in order to evaluate the neovascularization histopathologically [19]. In addition, CD34 is a marker that is bound to the surface of stem cells. In the literature, CD34 is frequently and safely evaluated in identifying stem cells [11]. VEGF and CD34 antibodies were used as markers in this study where tissue regeneration was investigated in the diabetic population using erythropoietin. Thus, the role of erythropoietin in stem cell synthesis and tissue regeneration in the context of damaged tissues was evaluated. In our study, reticulocytes and serum levels of CD34 and VEGF in different tissues of the cardiovascular system were evaluated in the diabetic model of rats for the first time in the literature.

In diabetic rats, CD34 and VEGF levels were higher in cardiovascular system tissues compared to healthy rats. The administration of erythropoietin, which is known to increase the amount of stem cells and endothelial growth factor, led to a larger increase in tissue levels of CD34 and VEGF in diabetic rats. Another remarkable finding is that although there was an increase in CD34 and VEGF levels and larger fibrosis reduction in the presence of erythropoietin in diabetics, it did not significantly correlate with reticulocyte levels, fibrosis, CD34 and VEGF levels except right atrial tissue. The current situation was thought to be related to the low number of subjects in the groups. A significant correlation was observed only in serum reticulocyte level and right atrium CD34 level.

The major limitation of our study is the low number of subjects. Also, we failed to detect a correlation between reticulocyte levels, CD34 and VEGF levels in different tissues of the heart other than the right atrium. Although the results indicated higher levels of CD34 and VEGF in erythropoietin induced diabetic rats, the provocative mechanism of erythropoietin to induce stem cells other than reticulocytes could not be clearly identified in this study.

In conclusion, the amount of CD34 and VEGF for tissue regeneration increases in the case of fibrotic insult in the cardiovascular system in rats. Tissue levels of CD34 and VEGF molecules that are involved in cellular protection and regeneration may be further enhanced by the safe use of erythropoietin in the diabetic rat population.

\section{Acknowledgement}

The study is associated with thesis of Didem Melis Oztas, MD and was supported by a grant from the BAPSIS of Istanbul University (Project Number: TTU-2018-31794).

\section{Conflict of interest}

The authors declare no conflict of interest.

\section{References}

1. Zaccardi F, Webb DR, Yates T, Davies MJ. Pathophysiology of type 1 andtype 2 diabetesmellitus: a 90-year perspective. Postgrad Med J 2016; 92: 63-9.

2. Goradel NH, Hour FG, Negahdari B, et al. Stem cell therapy: a new therapeutic option for cardiovascular diseases. J Cell Biochem 2018; 119: 95-104.

3. Dursunoğlu D, Evrengül H, Kaftan A, Kılıç M, Sermez Y. Koroner Ateroskleroz ve Diabet. Klinikleri J Cardiol 2004; 17: 55-60.

4. Nishimura H. Diabetes mellitus and atherosclerosis. Nihon Rinsho 2011; 69: 131-7.

5. Yenigün M. Kardiyovasüler Diabet. In: Her Yönüyle Diabetes Mellitus. Yenigün M, Altuntaş Y (eds). Nobel Tıp Kitabevleri, İstanbul 2001; 639-97.

6. Ugurlucan M, Yerebakan C, Furlani D, Ma N, Steinhoff G. Cell sources for cardiovascular tissue regeneration and engineering. Thorac Cardiovasc Surg 2009; 57: 63-73.

7. Furlani D, Ugurlucan M, Ong L, et al. Is the intravascular administration of mesenchymal stem cells safe? Mesenchymal stem cells and intravital microscopy. Microvasc Res 2009; 77: 370-6.

8. Packer C, Boddice B, Simpson S. Regenerative medicine techniques in cardiovascular disease: where is the horizon? Regen Med 2013; 8: 351-60.

9. Campbell NG, Suzuki K. Cell delivery routes for stem cell therapy to the heart: current and future approaches. J Cardiovasc Transl Res 2012; 5: 713-26.

10. Klopsch C, Furlani D, Gäbel R, et al. Intracardiac injection of erythropoietin induces stem cell recruitment and improves cardiac functions in a rat myocardial infarction model. J Cell Mol Med 2009; 13: 664-79.

11. Gäbel R, Klopsch C, Furlani D, et al. Single high-dose intramyocardial administration of erythropoietin promotes early intracardiac proliferation, proves safety and restores cardiac performance after myocardial infarction in rats. Interact Cardiovasc Thorac Surg 2009; 9: 20-5.

12. Ugurlucan M, Erer D, Kalko Y, et al. Aortic stiffness in diabetes mellitus - association with glutamine and heatshock protein 70 expression: a pilot study based on an experimental rodent model. Expert Opin Ther Targets 2009; 13: 267-74.

13. Lu J, Yao YY, Dai QM, et al. Erythropoietin attenuates cardiac dysfunction by increasing myocardial angiogenesis and inhibiting interstitial fibrosis in diabetic rats. Cardiovasc Diabetol 2012; 11: 105.

14. Park JY, Jeon HJ, Kim TY, et al. Comparative analysis of mesenchymal stemcell surface marker expression for human dental mesenchymal stem cells. Regen Med 2013; 8: 453-66.

15. Onat A. 'TEKHARF' ve 'Türk Kalp' Çalışmaları: Kapsam ve Katkılarına Objektif Bir Bakış. Türk Kardiyol Dern Arş 1994; 22: 10-1.

16. Teralı K, Ergören MÇ. The contribution of NOS3 variants to coronary artery disease: a combined genetic epide- 
miology and computational biochemistry perspective. Int J Biol Macromol 2018; 123: 494-9.

17. Yerebakan C, Sandica E, Prietz S, et al. Autologous umbilical cord blood mononuclear cell transplantation preserves right ventricular function in a novel model of chronic right ventricular volume overload. Cell Transplant 2009; 18: 855-68.

18. Ribatti D, Presta M, Vacca A, et al. Human erythropoietin induces a pro-angiogenic phenotype in cultured endothelial cells and stimulates neovascularization in vivo. Blood 1999; 93: 2627-36.

19. Bahlmann FH, De Groot K, Spandau JM, et al. Erythropoietin regulates endothelial progenitor cells. Blood 2004; 103: 921-6.

20. Cheng Y, Hu R, Lv L, Ling L, Jiang S. Erythropoietin improves the efficiency of endothelial progenitor cell therapy after myocardial infarction in mice: effects on transplanted cell survival and autologous endothelial progenitor cell mobilization. J Surg Res 2012; 176: e47-55.

21. Grundy SM, Benjamin IJ, Burke GL, et al. AHA Scientific Statement: diabetes and cardiovascular disease. A Statement for Health Care Professionals from the American Heart Association. Circulation 1999; 100: 1134-46. 